\title{
Determining the antioxidant compounds of beer
}

\author{
Loránd Alexa - Andrea Kántor - Béla Kovács - Nikolett Czipa \\ University of Debrecen, Institute of Food Science, Debrecen \\ alexal@agr.unideb.hu
}

\begin{abstract}
SUMMARY
The increasing consumption of beer indicates the necessity of the thorough analysis of its composition. In this study, the total phenolic content, flavonoid content and colour intensity of 24 beer samples have been determined. The samples contained pale barley, dark barley, pale wheat and dark wheat beers. The aim of the study was to determine the amount of the antioxidant compounds in beer, as well as to find correlation between different beer types and the above mentioned parameters.

Dark barley samples contained phenolic compounds in the highest concentrations, and it can also be concluded that dark beers contain phenolic compounds and flavonoids in higher concentrations than pale beers in the case of barley and wheat samples.

According to the performed statistical analysis, these beer types cannot be differentiated based on the analysed parameters. The highest percentage of correctly classified samples could be observed in case of dark barley samples, but this value has only been 75\%, and the total result of correctly classified cases was only $41.7 \%$. The analysis of more samples and parameters is required to carry out a successful differentiation.
\end{abstract}

Keywords: beer, TPC, flavonoid, colour, differentiation

\section{INTRODUCTION}

Beer is one of the most commonly consumed alcoholic beverages, which is produced from malt, hop and water, by adding yeasts. The quality of this drink can be influenced by the various brewing parameters (Gerhäuser 2005). The importance of high quality is verified by the fact that beer consumption is increasing, especially in countries with a long tradition of brewing, where this value has already reached 100 1/year/capita (FAO 2009). Using cereals as raw materials could provide natural antioxidant compounds, such as phenolic compounds and sulphites (Polak et al. 2013). Phenolic compounds are secondary metabolites of different plants. These substances are produced when the plant is exposed to stress conditions, and they play natural defensive role against pests and diseases (Oliveira et al. 2017). Flavonoids are a family of phenolic compounds, which can be found in significant concentrations mainly in fruits, vegetables, red wine and tea, and have high antioxidant capacity (Fusco et al. 2017). These antioxidant compounds could be extraordinary protecting factors in preventing oxidative stress, which can be responsible for conditions like Parkinson and Alzheimer's disease, cancer, and the aging process in general, which could be indicated by the increasing production of free radicals (Presti et al. 2017).

In this study, the total phenolic content (TPC), flavonoid content and colour intensity of 24 beer samples have been analysed. The aim was to determine whether a correlation between the antioxidant compounds, colour and the types of the cereals could be observed or not.

\section{MATERIAL AND METHODS}

\section{Samples and sample preparation}

24 beer samples were analysed, produced from barley (Hordeum vulgare convar. distichon) and wheat
(Triticum aestivum). The samples contained pale barley, dark barley, pale wheat and dark wheat beers. Samples could be originated from Hungary, The Czech Republic, Belgium, Germany and Austria.

To perform the measurements, samples needed to be decarbonized by using ultrasonic water bath (Bandelin Sonorex Digital DT 255H, Germany) and filtered through filter paper (Ahlstrom Munktell. Grade: 292. Stockholm, Sweden).

\section{Determination of colour intensity}

The prepared (decarbonized) sample has been poured into a cuvette, then its absorbance (A) has been measured at a wavelength of $430 \mathrm{~nm}$ with spectrophotometer (Evolution 300 LC, Thermo Electron Corporation, England). Colour intensity could be calculated using the following formula:

$$
\text { Colour intensity }=10 * \mathrm{~A}_{430}
$$

By applying this method, the results were measured on the SRM (Standard Reference Method) scale. To calculate the equivalent values on the EBC (European Brewing Convention) scale, results had to be multiplied by $1.97 \rightarrow \mathrm{EBC}=1.97 * \mathrm{SRM}(\mathrm{Net} 1)$.

\section{Determination of total phenolic content}

The principle of the measurement is the property of Folin-Ciocalteu reagent that it oxidizes phenolic compounds, which turns the solution to blue. The determination had been carried out according to the method of Signleton et al. (1999). In order to prepare the calibration solutions, gallic acid had been used, which is why results are reported in $\mathrm{mg} \mathrm{GAE} 100 \mathrm{ml}^{-1}$.

\section{Determination of flavonoid content}

The analysis has been performed according to the method of Kim et al. (2003). In order to prepare the calibration solutions, catechin had been used; therefore, the obtained results are reported in $\mathrm{mg} \mathrm{CE} 100 \mathrm{ml}^{-1}$. 


\section{Statistical analysis}

All analysis was performed in triplicate. Results were described by using general terms such as mean, standard deviation, minimum and maximum values. After the general description, Independent Samples Test and Linear Discriminant Analysis (LDA) were performed. For the statistical analysis SPSS for Windows (version 13, SPSS Inc. Chicago, Illinois, USA) has been used.

\section{RESULTS AND DISCUSSION}

\section{Colour Intensity}

The measured colour intensities of the analysed samples are shown in Table 1, containing the type and the origin of the samples. Results are shown on the SRM and the EBC scale. According to the directive no.2702 of Codex Alimentarius Hungaricus, beers could be divided into 3 groups due to their colour. These groups are the followings:

- pale beer (ale): $<20 \pm 3.0 \mathrm{EBC}$,

- semi-dark/red beer: $20-45 \pm 6.0$ EBC,

- dark beer: $>45 \pm 15.0$ EBC (Codex Alimentarius Hungaricus, 2013).

Colour intensity, type and origin of the analysed samples

\begin{tabular}{|c|c|c|c|c|}
\hline Sample & Type & Producer & SRM & EBC \\
\hline 1 & pale barley & Belgium & 5.89 & 11.6 \\
\hline 2 & pale barley & Hungary & 5.68 & 11.2 \\
\hline 3 & pale barley & Hungary & 3.60 & 7.09 \\
\hline 4 & pale barley & Hungary & 2.86 & 5.63 \\
\hline 5 & pale barley & Hungary & 4.75 & 9.35 \\
\hline 6 & pale barley & Czech Republic & 3.89 & 7.65 \\
\hline 7 & pale barley & Czech Republic & 6.32 & 12.4 \\
\hline 8 & pale barley & Hungary & 7.62 & 15.0 \\
\hline 9 & pale barley & Hungary & 4.20 & 8.27 \\
\hline 10 & dark barley & Belgium & 26.0 & 51.2 \\
\hline 11 & dark barley & Hungary & 32.4 & 63.8 \\
\hline 12 & dark barley & Czech Republic & 30.5 & 60.1 \\
\hline 13 & dark barley & Hungary & 42.0 & 82.8 \\
\hline 14 & dark barley & Czech Republic & 37.5 & 73.9 \\
\hline 15 & dark barley & Hungary & 38.5 & 75.8 \\
\hline 16 & dark barley & Czech Republic & 38.3 & 75.5 \\
\hline 17 & dark barley & Czech Republic & 36.2 & 71.4 \\
\hline 18 & pale wheat & Germany & 9.29 & 18.3 \\
\hline 19 & pale wheat & Germany & 6.28 & 12.4 \\
\hline 20 & pale wheat & Czech Republic & 8.15 & 16.1 \\
\hline 21 & pale wheat & Austria & 5.98 & 11.8 \\
\hline 22 & pale wheat & Germany & 7.47 & 14.7 \\
\hline 23 & dark wheat & Germany & 16.1 & 31.7 \\
\hline 24 & dark wheat & Germany & 29.3 & 57.7 \\
\hline
\end{tabular}

According to these results, the colours of the samples are in accordance with the requirements of the directive mentioned above. Difference from the nominal value could be observed only in the case of sample no. 23 (dark wheat), but this still has fallen within the tolerance limit.

\section{Total Phenolic Content (TPC)}

The total phenolic contents of the samples are shown in Figure 1. The obtained results showed wide ranges. The lowest concentration was $16.1 \mathrm{mg}$ GAE 100 $\mathrm{ml}^{-1}$ (sample no. 9., pale barley), and the highest concentration $\left(56.2 \mathrm{mg}\right.$ GAE $\left.100 \mathrm{ml}^{-1}\right)$ could be observed in a dark barley sample (13). Pale and dark wheat samples (18-24) contained phenolic compounds in similar concentrations, but in the case of barley samples, both pale and dark beers contained these components in various concentrations, and according to this figure, no correlations can be observed at first sight.

As Figure 2 shows, the group of dark barley samples had the highest mean value $(35.8 \mathrm{mg} \mathrm{GAE}$ $100 \mathrm{ml}^{-1}$ ) of total phenolic content. In the case of pale barley, pale wheat and dark wheat samples, the mean values of the obtained results were similar (24.2-25.2 mg GAE $100 \mathrm{ml}^{-1}$ ), which is about $69 \%$ of the value calculated for dark barley samples. These correlations between TPC and beer colour are in accordance with the available literature. For instance, a book chapter of Saura-Calixto et al. (2009) indicates that the phenolic compounds of lager beers range between 31 and $37 \mathrm{mg}$ $100 \mathrm{ml}^{-1}$, while dark beers contain about $47-57 \mathrm{mg}$ $100 \mathrm{ml}^{-1}$ of these substances. However, the exact values are higher than our results, the ratio is similar.

\section{Flavonoid content}

Flavonoid contents of the analysed samples can be observed in Figure 3. The results of this measurement also show wide ranges (4.20-29.6 mg CE $100 \mathrm{ml}^{-1}$ ). Sample no. 8. (pale barley) contained the lowest amount of flavonoids, but the highest concentration could be found in sample no. 13. (dark barley), just like in the case of TPC. Low flavonoid content $(7.0 \mathrm{mg} \mathrm{CE}$ $100 \mathrm{ml}^{-1}$ ) could be observed in sample no. 9. also. According to the TPC and flavonoid contents of the samples mentioned above, there is a correlation between the concentrations of these compounds. The ranges of the obtained results of pale and dark wheat samples are a bit higher than in the case of TPC, but except for sample no. 20 (pale wheat) and 24 (dark wheat), the results of these groups were balanced, which cannot be claimed in the case of barley samples.

According to the mean values of the analysed beer types presented in Figure 4, dark samples contained flavonoids in higher concentrations than pale samples in the case of barley and wheat samples, too. The highest flavonoid concentrations could be found in dark barley samples in general, such as the concentration of phenolic compounds and pale barley samples showed the lowest concentrations. The results of pale barley, pale wheat and dark wheat samples were not similar in this case, unlike when analysing TPC.

By using general terms, it could be declared that the mean values of TPC and flavonoid content in the case of barley and wheat samples were similar, but more significant difference could be observed in their colour intensity. To find statistically verified differences between the beer types according to the analysed parameters, an Independent Samples Test had been 
performed first. According to the P-value, which was lower than 0.05 in case of total phenolic content, the variance of the beer types was heterogeneous. We could also make the same statement by observing the results of flavonoid content and colour intensity, because P-values were lower than 0.05 by these parameters also. Although the group statistics showed homogenous variance in case of TPC and flavonoid content, the program calculated heterogeneous variance. The test verified that there was no significant difference between the two types, because P-values [Sig. (2-tailed)] were higher than 0.05 by all of the analysed parameters, so the differences between the mean values are due to only random effects. We were not able to verify any difference between the two types.

Figure 1: Total Phenolic Contents of the analysed samples

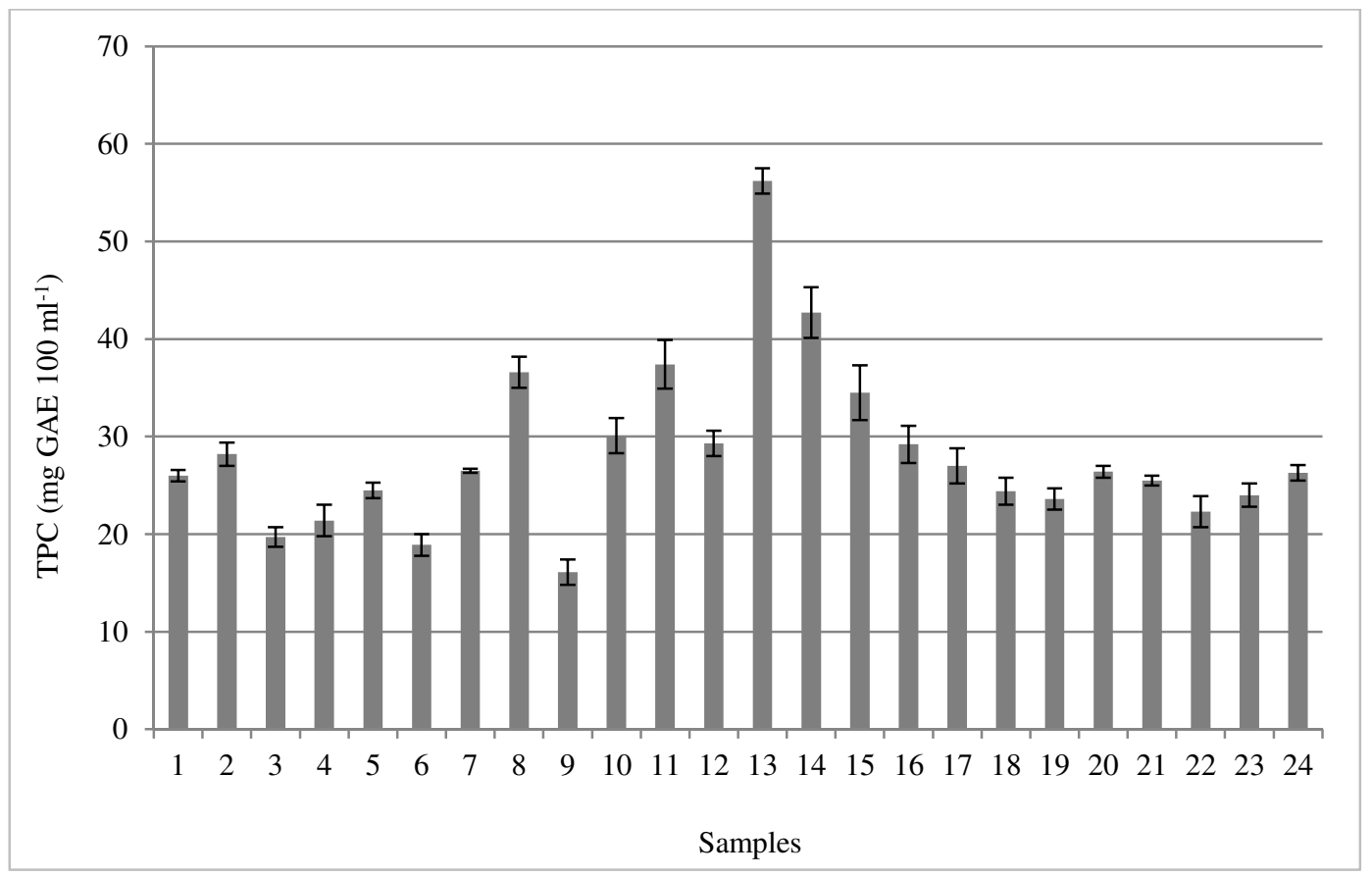

Figure 2: Total Phenolic Content of the analysed beer types

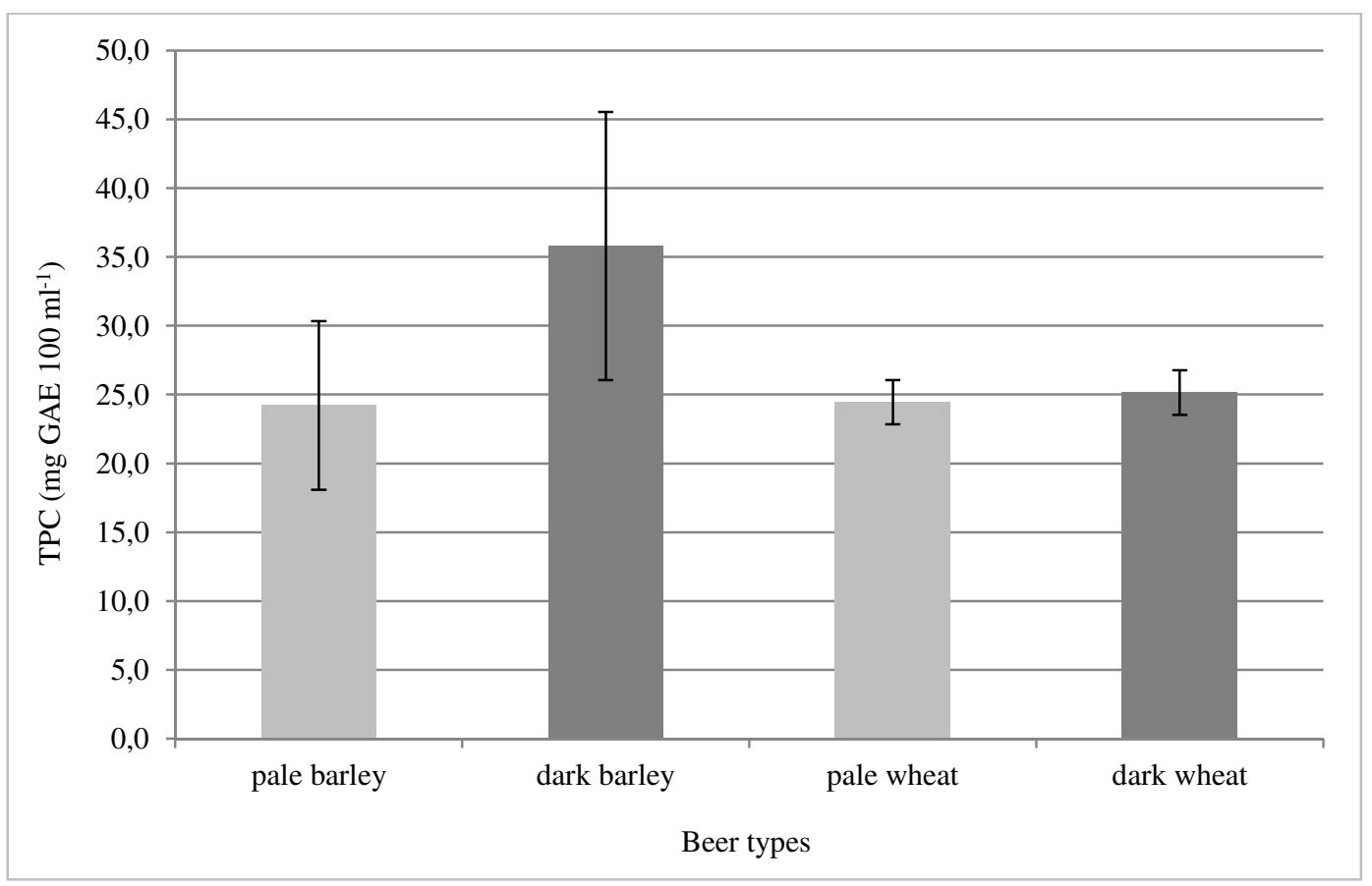




\section{Figure 3: Flavonoid contents of the analysed samples}

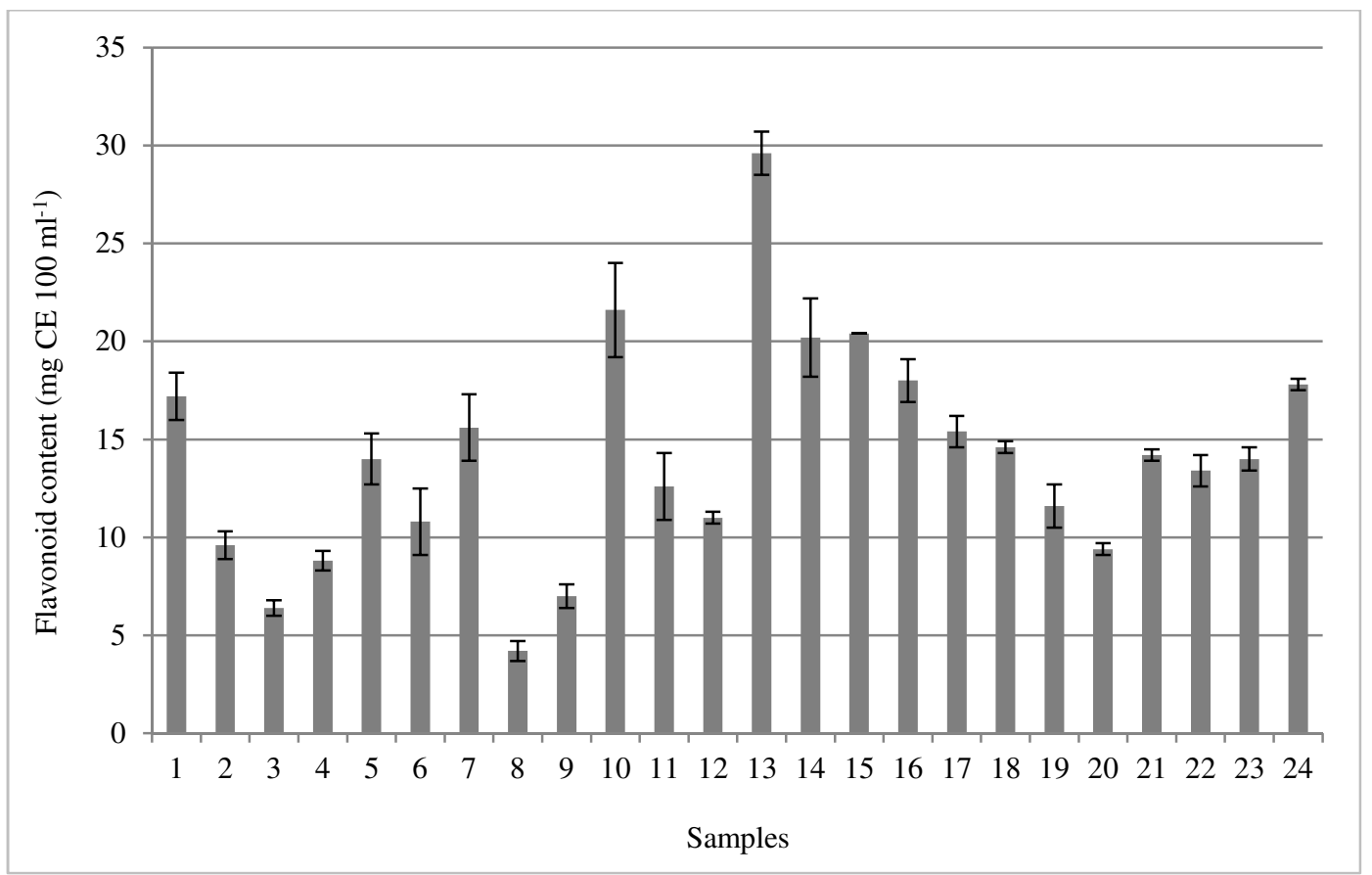

Figure 4: Flavonoid contents of the analysed beer types

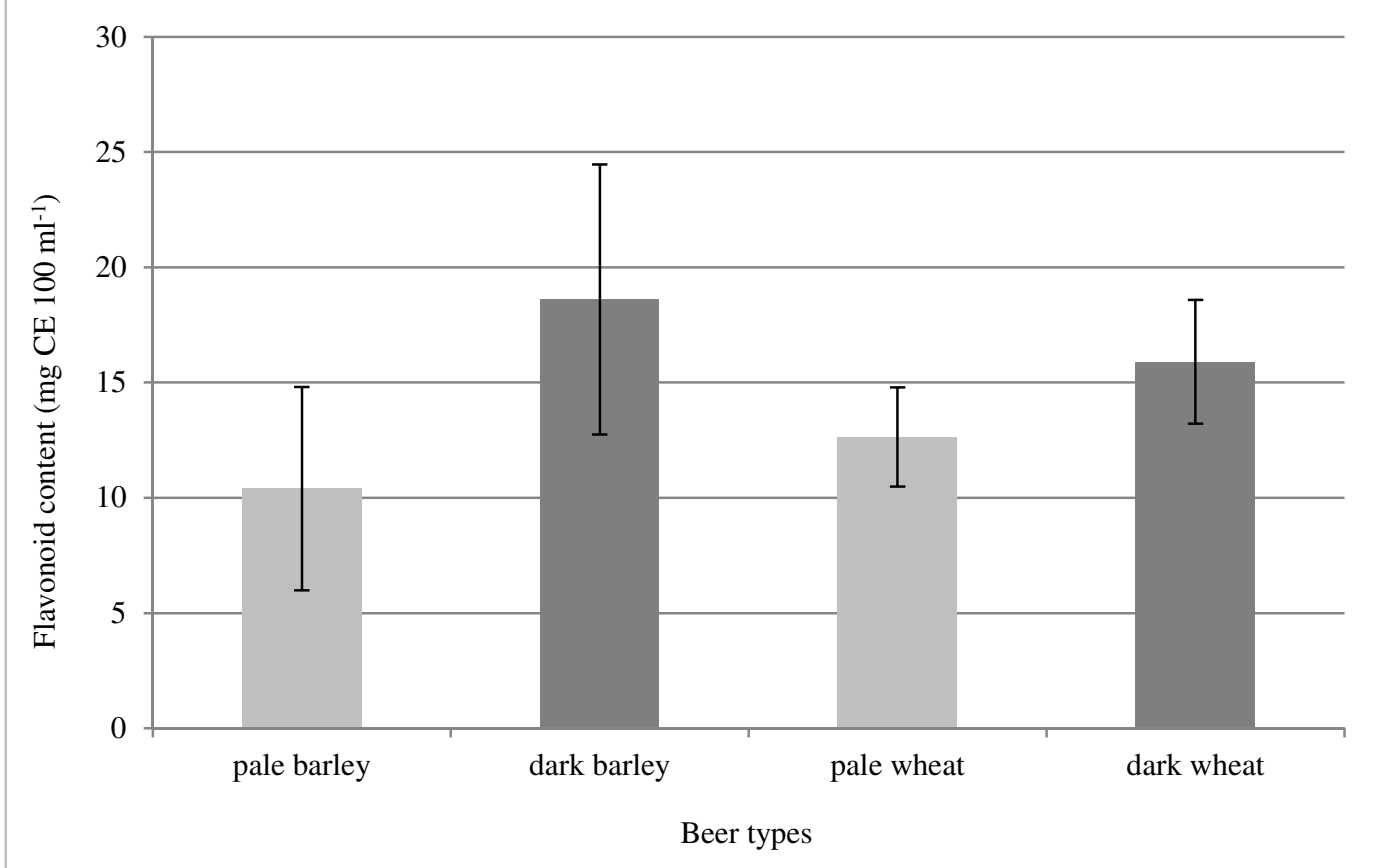

\section{Statistical analysis}

The correlation coefficient $\left(\mathrm{r}^{2}\right)$ of TPC and flavonoid content was 0.639 at the 0.01 level of significance. This coefficient of TPC and colour intensity was 0.695 , and $r^{2}$ was 0.690 in case of flavonoid content and colour intensity. This means that there is a correlation between the determined parameters, but this correlation is not strong.
To verify the distinction of different beer types, an LDA analysis has been carried out. The grouping variables were the beer types and the independent variables were the analysed parameters. Three discriminant functions were determined. The first function, which contained colour intensity, explained $52.6 \%$ of the variance of variables, the second one, which contained TPC, explained $5.50 \%$, and the third one contained flavonoid 
content and explained $0.16 \%$. This means that the first function, which contained the colour intensity, had the highest effect. Based on the Wilks' Lambda ratio, colour intensity had the greatest effect on the function, and this effect was significant. According to the eigenvalues, the first function was the most important, which have explained $52.6 \%$ of the variance of variables. The effect of the second and third function was not significant. The first and second dimensions are shown in Figure 5. As there could be observed, the group of dark barley has the highest group centroid
(1.34) in the first dimension. This value is -0.375 in case of pale wheat, -0.558 in case of dark wheat, and -0.861 by pale barley samples. In the second dimension, group centroids were really close to each other. Despite the similar group centroids in the second dimension, dark barley samples could be separated from the other groups in the first dimension.

Unfortunately, the differentiation of pale barley, pale wheat and dark wheat samples was not successful. To confirm this statement, we determined the percentage of correctly categorized samples.

Figure 5: Results of LDA analysis

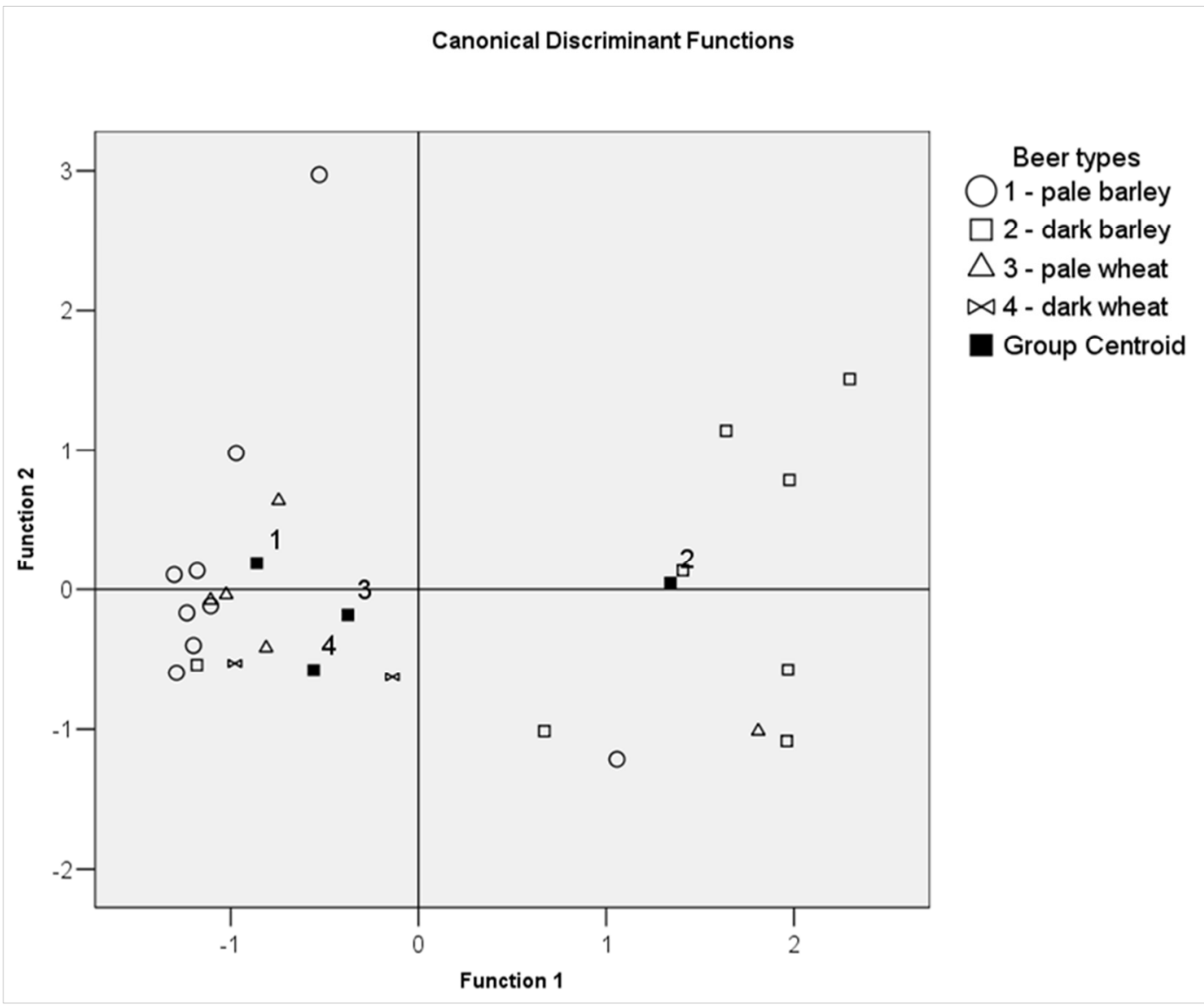

According to the performed cross validation, only $44.4 \%$ of pale barley samples had been classified correctly, because 2 samples moved into the group of dark barley, and 3 samples moved into the group of dark wheat samples. In the case of dark barley samples, this value has reached 75\%. 6 samples were correctly categorized, but 1 sample moved into the group of pale barley, and 1 sample moved into the group of dark wheat samples. None of the samples could be found in their own group in the case of pale and dark wheat samples, the determined value was $0 \%$. Overall, only $41.7 \%$ of cross-validated grouped cases were correctly classified; therefore, it can be declared that in the case of the analysed samples, the determination of these parameters has not been suitable for the differentiation of these beer types.

\section{CONCLUSION}

In the present paper, 24 beer samples have been analysed, which contained pale barley, dark barley, pale wheat and dark wheat samples. Total phenolic contents and flavonoid contents have been determined to shed light on the antioxidant properties of beer, and we have tried to find correlations between colour, raw materials and antioxidant compounds.

It turned out that dark barley samples contained phenolic compounds in the highest concentration, while TPC showed similar values in case of pale barley, pale wheat and dark wheat samples. A higher concentration of flavonoids can also be observed in dark samples than in pale beers by barley and wheat samples too, 
which suggests that the method of malting could influence TPC and flavonoid content.

According to the performed statistical analysis, these beer types cannot be differentiated according to the determined parameters. Only $41.7 \%$ of cross-validated grouped cases were correctly classified, which is a poor result. We could reach a higher value in the case of the differentiation of dark barley samples, but this value still was not high enough. To carry out a successful differentiation, the determination of more parameters (e.g. element contents) and more samples would be recommended.

\section{REFERENCES}

Codex Alimentarius Hungaricus (2013): Beer. 3. 3. Directive no. $2-$ 702

FAO (2009): Malt, Barley, Beer. Agribusiness Handbook. Rome. Italy.

Fusco, R.-Cirmi, S.-Gugliandolo, E.-Di Paola, R.-Cuzzocrea, S.Navarra, M. (2017): A flavonoid-rich extract of orange juice reduced oxidative stress in an experimental model of inflammatory bowel disease. Journal of Functional Foods. 30: $168-178$.

Gerhäuser, C. (2005): Beer Constituents as Potential Cancer Chemopreventive Agents. European Journal of Cancer. 41: 1941-1954.

Kim, D. O.-Jeong, S. W.-Lee, C. Y. (2003): Antioxidant Capacity of Phenolic Phytochemicals from Various Cultivars of Plums. Food Chemistry. 81: 321-326.

Net1: Beer Color: Understanding SRM, Lovibond and EBC. http:// beersmith.com/blog/2008/04/29/beer-color-understanding-srmlovibond-and-ebc/ 2017. 11. 02.
Oliveira, K. G.-Queiroz, V. A. V.-Carlos, L. A.-Cardoso, L. M.Pinheiro-Sant'Ana, H. M.-Anunciacao, P. C.-Menezes, C. B.Silva, E. C.-Barros, F. (2017): Effect of the storage time and temperature on phenolic compounds of sorghum grain and flour. Food Chemistry. 216: 390-398.

Polak, J.-Bartoszek, M.-Stanimirova, I. (2013): A Study of the Antioxidant Properties of Beers Using Electron Paramagnetic Resonance. Food Chemistry. 141: 3042-3049.

Presti, G.-Guarrasi, V.-Gulotta, E.-Provenzano, F.-Giuliano, S.Monfreda, M.-Mangione, M. R.-Passantino, R.-San Biagio, P. I.-Costa, M. A.-Giacomazza, D. (2017): Bioactive Compounds From Extra Virgin Olive Oils: Correlation Between Phenolic Content and Oxidative Stress Cell Protection. Biophysical Chemistry. 230: 109-116.

Saura-Calixto, F.-Serrano, J.-Pérez-Jiménez, J. (2009): What Contribution Is Beer to the Intake of Antioxidants in the Diet? Beer in Health and Disease Prevention.

Singleton, V. L.-Orthofer, R.-Lamuela-Raventos, M. (1999): Analysis of Total Phenols and Other Oxidation Substrates and Antioxidants by Means of Folin-Ciocalteu Reagent. Methods in Enzymology. 299: 152-178. 